\title{
RADIOGRAPHIC ASSESSMENT OF DIGITAL TRAY TECHNIQUE VERSUS CONVENTIONAL TECHNIQUE ON THE ACCURACY OF MANDIBULAR SCREW RETAINED IMPLANT OVERDENTURES
}

\author{
Aya Mohamed Fawzy Hafez * and Shady M. El-Naggar**
}

\begin{abstract}
Purpose: This study purposed to be introduced to evaluate the effect of digital tray technique used on the accuracy of mandibular screw-retained implant overdenture compared with complete denture incorporated with barium sulphate as radiographic stent.

Materials and Methods: Forty-eight patients were examined for implant placement. Each implant site was planned virtually using two different techniques: denture duplication technique with barium sulphate incorporation and digital tray technique. Implantation placement had been performed in both groups using the same three dimensional manufactured radiographic stent. Post insertion CBCT was introduced with simulated scheduling images. Angular deviation, global deviation, lateral deviation and depth of implant placement were collected for planned and placed implants of both groups.
\end{abstract}

Results: Using independent t-test for significance assessment of global, lateral and angular deviation between both groups, it was revealed that there was significant difference between both groups and insignificant difference for implant depth.

Conclusion: Within limitations regarding radiographic accuracy depending on radiographic technique; it showed less amount of deviation of digital tray group comparing to conventional denture duplication technique with barium sulphate incorporation.

KEYWORDS: Digital, Implant, Accuracy, Denture, Radiograph

\section{INTRODUCTION}

Dental implants had become a treatment modality generally used for the restoration of missing teeth. The introduction of dental implants had a significant effect on the people and the implant supported prosthesis had become a widely used treatment modality for edentulous patients. ${ }^{1}$

Advancement in oral implantology can no longer be limited to osseointegration and maintenance of alveolar bone. Osseointegration has shown success

\footnotetext{
* Department of Removable Prosthodontics, Faculty of Oral and Dental Medicine, Future University in Egypt, Egypt. ** Department of Removable Prosthodontics, Faculty of Oral and Dental Medicine, Badr University in Cairo, Egypt.
} 
and high predictability in the literature repeatedly. ${ }^{2}$ Another principle is implant insertion that allow introduction of a restoration with a pleasing appearance. Proper placement is among the critical factors in implant dentistry. ${ }^{3}$ Prosthetically Driven, Computer-Guided Implant Planning is our main goal nowadays, which advances the predictability of the treatment, permits for a better risk management, and delivers more individual information for the patient. ${ }^{4}$

Placement of implants has been introduced and assisted by means of simple periapical and panoramic radiographs for years. ${ }^{5}$ However, surgical models have achieved higher role of interest as resources to accomplish higher accurate fixture insertion in the operatory field due to both the prosthetic strategy and the internal anatomy needed to be considered ${ }^{6}$

Traditionally, 2-D sections and a model constructed on the dental cast as a traditional surgical guide, were used to support adequate surgical insertion. Lately, CBCT had achieved approval and is frequently used in the virtual process. ${ }^{7}$

Computer-assisted design/computer-aided manufacturing (CAD/CAM) allows the production of surgical guides that are virtually planned and designed using data accrued from 3D imaging utilizing computer software and digital workflow for planning and manufacturing. They have metallic sleeves that permit accurate fixture insertion in different axis. Scanning and digital imaging ways, which allow imagining of insertion of dental implants in different dimensions, have added attractiveness in their uses given their ability to accomplish liable and precise outcomes. ${ }^{8}$

Usage of such a system frequently eliminates difficulties including, sinus perforations mandibular nerve damage, fenestrations or dehiscence. The dentist can mimic proper fixture insertion and treatment visualization that includes the precise dimensions of the implant, the ideal depth, and angulation made on the CBCT scans. In addition, this improved precision and accuracy reduces the need for flap reflection. ${ }^{9}$

CAD/CAM uses different approaches for manufacturing, subtractive manufacturing is usually accomplished by the Computer Numerical Controlled (CNC) machining, creating objects by progressive removal or deformation of material (drilling, cutting, bending) from a large block or sheet in the form of chips. ${ }^{10}$ While additive manufacturing (3D printing) is a manufacturing method in which objects are made by fusing or depositing materials - for example metal, plastic, ceramics, liquids, powders or even living cells - in layers to produce a $3 \mathrm{D}$ object ${ }^{11}$.

Modern dentistry has advanced from cone-beam computed tomography (CBCT), intra-oral scanners, computer-aided design (CAD) software, and computer-aided manufacturing (CAM), which has had a reflective influence on implant dentistry. In specific, additive manufacturing (AM) technology, known as $3 \mathrm{D}$ printing, has contributed to the effective application of computer-guided implant surgery. ${ }^{12,13}$ Traditional implant surgical guides with modified radiographic templates necessitate complicated laboratory procedures which can be imprecise, resulting in difficulty in accurate positioning of the implants in their planned position ${ }^{14}$. In contrast, computer-assisted implant surgical guides can avoid endangering vital anatomical structures, save time, and assist implant placement by drawing the preplanned final prosthesis design ${ }^{15,16}$

Implant planning $\mathrm{CAD}$ software; to process the information obtained from CBCT, together with intra-oral scanners, and diagnostic casts are used to design precise and accurate computer-assisted implant surgical guides. ${ }^{17,18}$ A systemic review measured the accuracy of the implant surgical guides designed using different implant planning CAD software, the results presented that the mean apical deviation between the virtual position and placed position was $1.4 \mathrm{~mm}$, and the angular deviation was 
3.5. ${ }^{19}$ Though, these deviations may vary among different studies ${ }^{20}$

Another study showed that the positioning accuracy of computer-guided implants can differ depending on several experimental factors. The accuracy of a cone beam computed tomogram (CBCT) or intraoral scanning of the patient, and the accuracy of $3 \mathrm{D}$ printing or milling, to have a significant role on accurate implants positioning. ${ }^{21}$ If the positioning accuracy of computer-guided implants is inaccurate, it will result in unplanned implant position, which may be the major cause of its failure.

Accuracy can be measured by superimposing the pre- and post-operative CBCT images for the placed implant. Unfortunately many errors could result as inaccuracy of $\mathrm{CBCT}$ due to resolution and distortion, faulty superimposition of the CBCT images. Furthermore, the existence of metal artifacts in the oral cavity may decreases the resolution of the CBCT images ${ }^{22,23}$ Recently a nonradiographic method has been introduced by using an intraoral scanner that allows superimposing the placed implant position on the previously obtained implant fixture scan data ${ }^{24}$. Which offers accurate for assessment of the placed implant and saves postoperative CBCT imaging.

The stereolithography apparatus (SLA) that could create 3D models from digital data was initially introduced by Charles Hull as an early 3D printing device. ${ }^{25}$ The 3D-printer market is further developed resulting, in smaller 3D printers that are being introduced into dental clinics for quicker results and lower costs known by in-once or personal $3 \mathrm{D}$ printers ${ }^{26-30}$. Computer-assisted implant surgical guides can be easily produced with personal 3D printers after being designed with implant planning CAD software. ${ }^{31}$

In spite of the accuracy of computer-assisted implant guides produced by personal 3D printers is a significant issue in their clinical practice, there is still a deficiency of research investigating their accuracy. Consequently, this study assessed the accuracy of computer-assisted implant surgical guides, which were designed using implant planning CAD software (Deltanine and R2gate software) and manufactured through personal 3D printers utilizing a non-radiographic technique. The null hypothesis of this study is that there is no difference in the accuracy of surgical guides fabricated by both types of software. ${ }^{32}$

\section{Sample Size Calculation}

Sample size calculation of this randomized controlled trial was calculated based on Ahmed et al $2019^{33}$, If the true difference in the experimental and control means is 1 , we will need to study 48 subjects (24 in each group) to be able to reject the null hypothesis that the population means of the experimental and control groups are equal with probability (power) 0.8 . The Type I error probability associated with this test of this null hypothesis is 0.05 .

\section{MATERIALS AND METHODS}

This study was a randomized clinical trial (RCT), using cone-beam computed tomography (CBCT) imaging and flapless surgical technique to place four mandibular implants under approval of Medical Ethical Committee of Future University; FUE-REC (8)/2-2021.

The study was performed on forty-eight completely edentulous male patients from the outpatient Clinic of Faculty of Oral and Dental Medicine, Future University, Department of Removable Prosthodontics and randomly divided according to radiographic technique into two groups;

Group (I); fully edentulous mandibular arches preoperatively scanned using duplicated conventional complete denture incorporated with barium sulphate. 
Group (II); fully edentulous mandibular arches preoperatively scanned using digital tray (Ray Co. Ltd., Korea).

Each patient received a screw-retained prosthesis; however, this study was mainly concerned with the mandibular arch. Each patient in this study was evaluated regarding the implant insertion accuracy of the edentulous area pre and post implant insertion.

The study was reviewed and approved by Ethics Committee in Faculty of Oral and Dental Medicine, Future University, Egypt.

The steps of conventional complete denture construction started by making primary alginate impressions, final impressions were done using border molded acrylic resin special tray and condensation silicone rubber base, jaw relation registration using face bow record and wax wafer technique, mounting and setting up of cross linked acrylic teeth on a semi adjustable articulator, laboratory remounting and finally denture delivery and post insertion adjustments were done.

After denture adjustment, the upper and lower denture were taken from the patient for duplication using laboratory duplication silicon (putty consistency). The duplication was performed using special plastic mold. Suitable amount of putty material was used to fill the lower compartment of the mold. While, still soft the denture was embedded from its polished surface so that the borders of the denture flush with the putty material. To have an imprint of the polished surface of both upper and lower dentures.

Then a separating medium was painted all over the set putty surface to facilitate separation from the next mix of putty in the other half of the mold. A second mix of putty material was prepared to fill the other half of the mold, which was closed under pressure until complete closure. After the setting, the plastic mold was opened and the denture was removed from the container.
Auto polymerizing acrylic resin powder was then properly mixed with barium sulphate powder (ratio 4:1. Total of $100 \mathrm{gm}$ ). Small holes were done (approximately $1 \mathrm{~mm}$ in diameter) in the central position of each tooth.

Then the stents were checked intraorally for stability and fitness. Any pressure area was relieved to allow complete seating. Now the radiographic stents were ready to use.

The templates were placed intraorally and the patient was asked to close, in centric position in higher vertical dimension, on a mix of putty silicone impression material. After setting, the occlusion record was removed from the patient's mouth and the excess material was removed from the holes to allow for reproducibility.

Regarding digital trays, putty consistency impression was supplied using such trays with corresponding suitable size.

The patient was asked to wear the radiographic templates and close on the putty record during imaging. The patient was asked to stay still till the machine rotates around him. Planning was done using specialized software (On Demand 3D App.), the obtained STL files were imported into it. The proposed implant sites were the canine and the second premolar (bilaterally). These proposed sites were identified by the radiolucent channels, previously prepared in the radiographic templates. The proposed implant diameter was $3.75 \mathrm{~mm}$ and the proposed length was $10 \mathrm{~mm}$ and $12 \mathrm{~mm}$ in the posterior and anterior areas respectively.

The desired implant sites were identified through the radiolucent channels previously prepared/ supplied in the radiographic stent at the prosthetic teeth center. The implants angle, global deviation, lateral deviation and depth at each of the four potential sites were evaluated for planned and placed implants. 




Fig. (1): Conventional radiographic stent using duplicated complete denture incorporated with barium sulphate

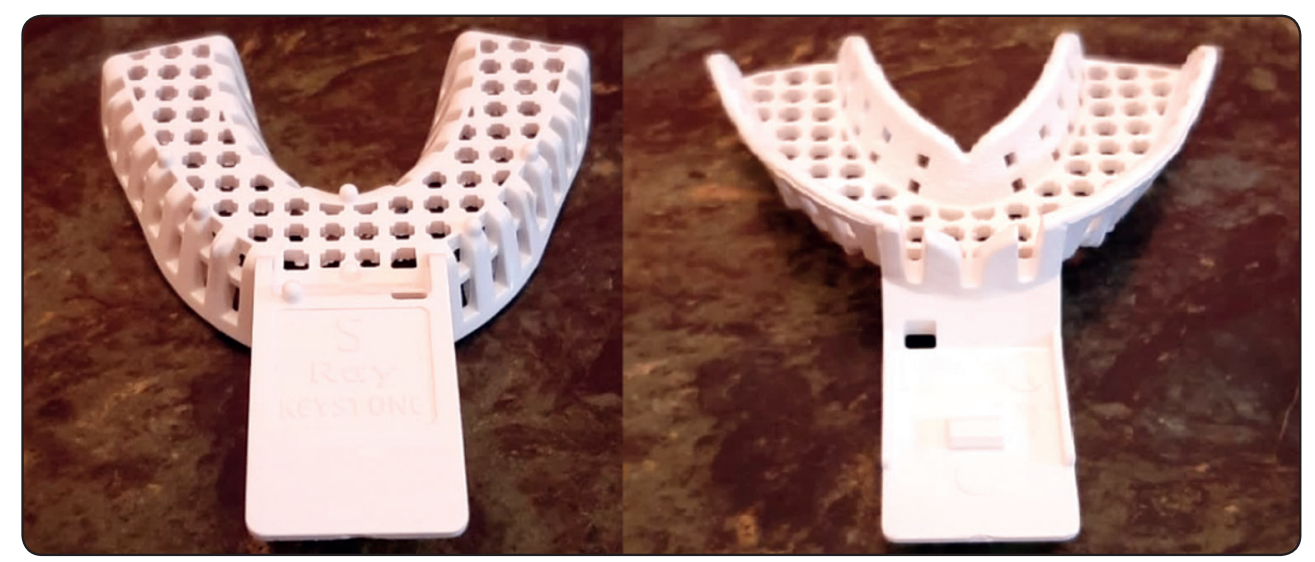

Fig. (2): Digital Tray
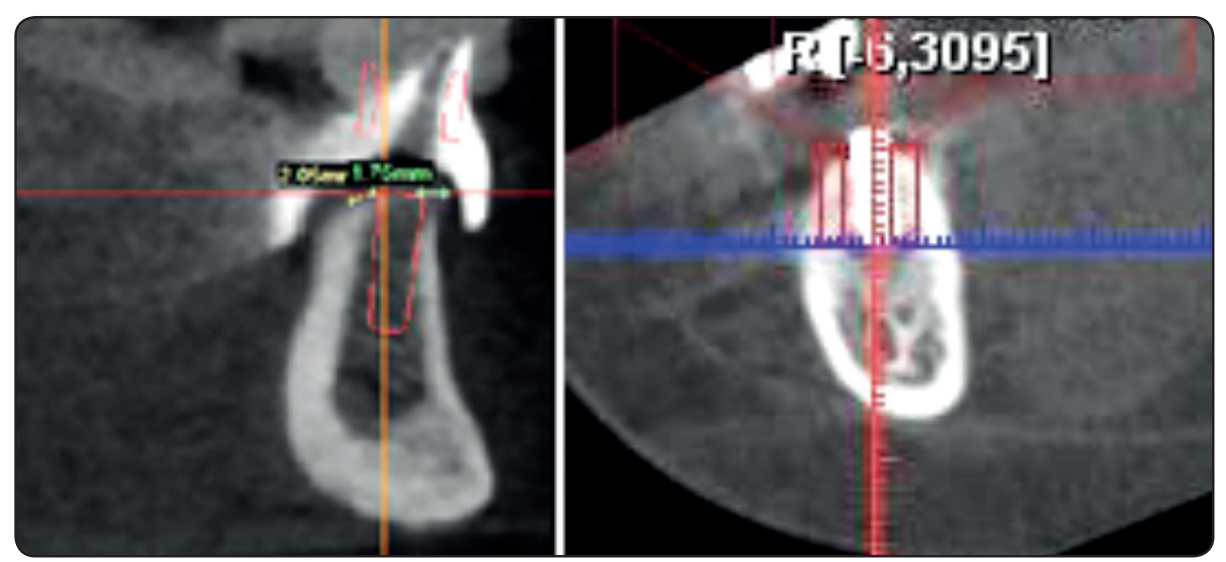

Fig. (3): Accuracy Parameters Determination of Planned and placed implants using CBCT 


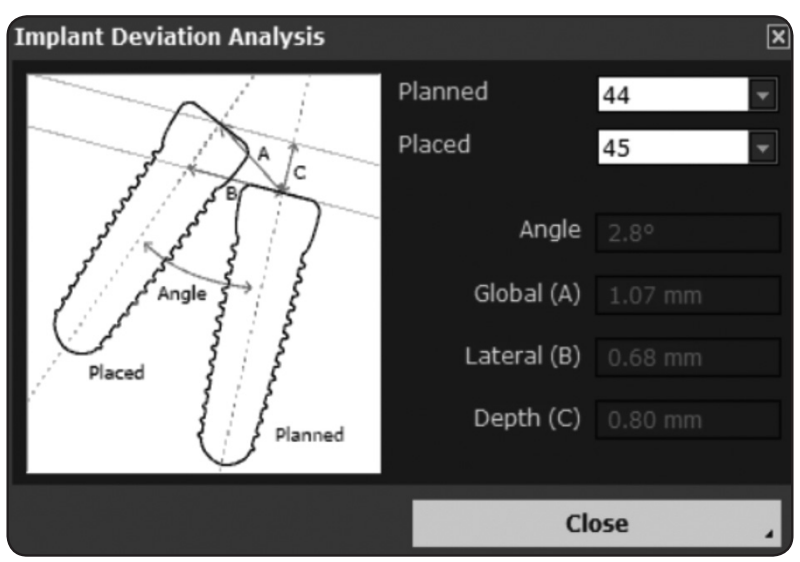

Fig. (4): Accuracy measurement using (On-demand 3D) software

The preoperative (implant planning) and postoperative (achieved implant position) scans were then overlapped using a dedicated algorithm, which allowed the comparison of the virtually planned and the actual implant positions. Four parameters between each planned and placed implant were measured. All measurements were performed using dedicated software (OnDemand3D, Cybermed Inc., Seoul, Korea).

For analyzing the accuracy, the planned position of the implant was compared with the actual position of the implant after insertion. Several measuring points were used for the comparison of these positions: ${ }^{34}$

1- The global deviation is the spatial distance between the centers of the implant platform/apex of virtual (planned) and final placed implants.

2- The lateral deviation is the directional component of the global deviation at the level of the virtual implant platform/apex.

3- The depth deviation is the distance of virtual and final placed implants on the axis of the virtual implant.

4- The angular deviation is the spatial angle between the virtual and final implant placements.
Though several methods were used to describe the distance between the given points, the most common method was to measure the actual distance between the planned and actual point in the $\mathrm{x}, \mathrm{y}$, and $\mathrm{z}$-axis, where $\mathrm{x}=$ bucco-lingual, $\mathrm{y}=$ mesio-distal, and $\mathrm{z}=$ apico-coronal deviation. The 3D deviation was calculated by the software using Pythagorean Theorem (21).

Statistical analysis performed with SPSS $20^{\circledR}$, Graph Pad Prism ${ }^{\circledR}$ and Microsoft Excel 2016. Data presented as means and standard deviation (SD) values in $\left(\right.$ Degrees $\left.^{\circ}\right)$ and $(\mathrm{mm})$.

\section{RESULTS}

For global deviation of the four planned and placed implants (Group I, duplicated conventional complete denture incorporated with barium sulphate), mean \pm standard deviation was revealed in MD section as $(1.8 \pm 0.56),(0.11 \pm 0.03)$, $(2.47 \pm 0.76)$ and $(2.02 \pm 0.62)$ with over all MD global deviation $(1.60 \pm 0.48)$, as listed in table (1) and showed in figure (6). While in BL section, it was revealed as $(1.2 \pm 0.37),(1.15 \pm 0.36),(1.19 \pm 0.37)$ and $(1.17 \pm 0.36)$ with over all BL global deviation $(1.18 \pm 0.36)$, as listed in table (1) and showed in figure (6).

For the four planned and placed implants (Group II, digital tray), mean \pm standard deviation was revealed in MD section as (1.12 \pm 0.35$)$, $(0.0379 \pm 0.01),(1.3 \pm 0.4)$ and $(2 \pm 0.62)$ with over all MD global deviation (1.11 \pm 0.34$)$, as listed in table (1) and showed in figure (6). While in BL section, it was revealed as $(1.09 \pm 0.35),(1.05 \pm 0.34)$, $(1.13 \pm 0.36)$ and $(1.13 \pm 0.36)$ with over all BL global deviation $(1.10 \pm 0.33)$, as listed in table (1) and showed in figure (6).

Using independent $t$ test for significance assessment of global deviation between both groups, it was revealed that there was significant difference between both groups regarding implant (1), (2), (3) and overall implants in MD section as 
P-value $<0.05$, as listed in table (1). On the other hand, there was insignificant difference between both groups in BL section for all implants as P-value $>0.05$, as listed in table (1)

For lateral deviation of the four planned and placed implants (Group I, duplicated conventional complete denture incorporated with barium sulphate), mean \pm standard deviation was revealed in $\mathrm{MD}$ section as $(0.657 \pm 0.18),(0.687 \pm 0.21)$, $(0.645 \pm 0.18)$ and $(0.693 \pm 0.21)$ with over all MD lateral deviation $(0.67 \pm 0.2)$, as listed in table (2) and showed in figure (7). While in BL section, it was revealed as $(0.843 \pm 0.26),(0.818 \pm 0.25)$, $(0.758 \pm 0.23)$ and $(0.837 \pm 0.26)$ with over all $\mathrm{BL}$ lateral deviation $(0.81 \pm 0.24)$, as listed in table (2) and showed in figure (7).

For the four planned and placed implants (Group II, digital tray), mean \pm standard deviation was revealed in MD section as $(0.615 \pm 0.19),(0.686 \pm 0.19)$, $(0.581 \pm 0.18)$ and $(0.679 \pm 0.19)$ with over all MD lateral deviation $(0.64 \pm 0.19)$, as listed in table (2) and showed in figure (7). While in BL section, it was revealed as $(0.532 \pm 0.15),(0.582 \pm 0.16),(0.521 \pm 0.14)$ and $(0.491 \pm 0.14)$ with over all BL lateral deviation $(0.53 \pm 0.16)$, as listed in table (2) and showed in figure (7).

Using independent $t$ test for significance assessment of lateral deviation between both groups, it was revealed that there was significant difference between both groups regarding implant (1), (2), (3), (4) and overall implants in BL section as P-value < 0.05 , as listed in table (2). On the other hand, there was insignificant difference between both groups in MD section for all implants as P-value $>0.05$, as listed in table (2).

For depth of the four planned and placed implants (Group I, duplicated conventional complete denture incorporated with barium sulphate), mean \pm standard deviation was revealed in MD section as $(0.841 \pm 0.21),(0.921 \pm 0.28),(0.808 \pm 0.20)$ and $(0.92 \pm 0.28)$ with over all MD depth $(0.83 \pm 0.25)$, as listed in table (3) and showed in figure (8). While in BL section, it was revealed as $(1.01 \pm 0.31)$, $(0.793 \pm 0.23),(0.843 \pm 0.26)$ and $(0.917 \pm 0.28)$ with over all BL depth $(0.87 \pm 0.26)$, as listed in table (3) and showed in figure (8).

For the four planned and placed implants (Group II, digital tray), mean \pm standard deviation was revealed in MD section as $(0.821 \pm 0.25)$, $(0.808 \pm 0.20),(0.797 \pm 0.25)$ and $(0.836 \pm 0.21)$ with over all MD depth $(0.73 \pm 0.22)$, as listed in table (3) and showed in figure (8). While in BL section, it was revealed as $(0.865 \pm 0.25),(0.732 \pm 0.23)$, $(0.712 \pm 0.21)$ and $(0.832 \pm 0.24)$ with over all $\mathrm{BL}$ depth $(0.82 \pm 0.25)$, as listed in table (3) and showed in figure (8).

Using independent $\mathrm{t}$ test for significance assessment of the depth between both groups, it was revealed that there was insignificant difference between both groups regarding all implants as $\mathrm{P}$-value $>0.05$, as listed in table (3). In addition, there was insignificant difference between both groups in MD section for all implants as P-value > 0.05 , as listed in table (3).

For angular deviation of the four planned and placed implants (Group I, duplicated conventional complete denture incorporated with barium sulphate), mean \pm standard deviation was revealed in MD section as $(4.52 \pm 1.4),(8.17 \pm 2.52)$, $(6.37 \pm 1.97)$ and $(5.01 \pm 1.55)$ with over all $\mathrm{MD}$ angular deviation $(6.02 \pm 1.82)$, as listed in table (4) and showed in figure (9). While in BL section, it was revealed as $(4.86 \pm 1.46),(7.36 \pm 2.21),(7.98 \pm 2.4)$ and $(4.59 \pm 1.38)$ with over all BL angular deviation (6.20 \pm 1.87$)$, as listed in table (4) and showed in figure (9).

For the four planned and placed implants (Group II, digital tray), mean \pm standard deviation was revealed in MD section as $(2.13 \pm 0.55),(4.78 \pm 1.24)$, $(3.46 \pm 0.9)$ and $(4.21 \pm 1.09)$ with over all MD angular deviation $(3.65 \pm 1.1)$, as listed in table (4) and showed in figure (9). While in BL section, it was 
revealed as $(2.34 \pm 0.72),(4.09 \pm 1.26),(4.57 \pm 1.41)$ and $(3.4 \pm 1.05)$ with over all BL angular deviation $(3.60 \pm 1.09)$, as listed in table (4) and showed in figure (9).

Using independent $t$ test for significance assessment of lateral deviation between both groups, it was revealed that there was significant difference between both groups regarding implant (1), (2), (3), (4) and overall implants in BL section as P-value $<0.05$, as listed in table (2). In addition, there was significant difference between both groups in MD section for all implants as $\mathrm{P}$-value $<0.05$, as listed in table (4).

TABLE (1): Global deviation evaluation between both groups regarding all implants and overall implants:

\begin{tabular}{|c|c|c|c|c|c|c|c|c|c|c|}
\hline & \multicolumn{10}{|c|}{ Global Deviation $(\mathbf{M} \pm \mathrm{SD})$} \\
\hline & \multicolumn{2}{|c|}{ Implant (1) } & \multicolumn{2}{|c|}{ Implant (2) } & \multicolumn{2}{|c|}{ Implant (3) } & \multicolumn{2}{|c|}{ Implant (4) } & \multicolumn{2}{|c|}{ Overall } \\
\hline & MD & $\mathbf{B L}$ & MD & BL & MD & BL & MD & $\mathbf{B L}$ & MD & $\mathbf{B L}$ \\
\hline Group (I) & $1.8 \pm 0.56$ & $1.2 \pm 0.37$ & $0.11 \pm 0.03$ & $1.15 \pm 0.36$ & $2.47 \pm 0.76$ & $1.19 \pm 0.37$ & $2.02 \pm 0.62$ & $1.17 \pm 0.36$ & $1.60 \pm 0.48$ & $1.18 \pm 0.36$ \\
\hline Group (II) & $1.12 \pm 0.35$ & $1.09 \pm 0.35$ & $0.0379 \pm 0.01$ & $1.05 \pm 0.34$ & $1.3 \pm 0.4$ & $1.13 \pm 0.36$ & $2 \pm 0.62$ & $1.13 \pm 0.36$ & $1.11 \pm 0.34$ & $1.10 \pm 0.33$ \\
\hline P-value & $0.0001 * *$ & $0.2955^{*}$ & $0.0001 * *$ & $0.3277 *$ & $0.0001 * *$ & $0.5719 *$ & $0.9115^{*}$ & $0.7012 *$ & $0.0002 * *$ & $0.0775 *$ \\
\hline
\end{tabular}

M; Mean, SD; Standard Deviation, P; Probability Level

MD; Mesiodistal, BL; Buccolingual

*insignificant difference

TABLE (2): Lateral deviation evaluation between both groups regarding all implants and overall implants:

\begin{tabular}{|c|c|c|c|c|c|c|c|c|c|c|}
\hline & \multicolumn{10}{|c|}{ Lateral Deviation $(\mathrm{M} \pm \mathrm{SD})$} \\
\hline & \multicolumn{2}{|c|}{ Implant (1) } & \multicolumn{2}{|c|}{ Implant (2) } & \multicolumn{2}{|c|}{ Implant (3) } & \multicolumn{2}{|c|}{ Implant (4) } & \multicolumn{2}{|c|}{ Overall } \\
\hline & MD & BL & MD & BL & MD & BL & MD & BL & MD & BL \\
\hline Group (I) & $0.657 \pm 0.18$ & $0.843 \pm 0.26$ & $0.687 \pm 0.21$ & $0.818 \pm 0.25$ & $0.645 \pm 0.18$ & $0.758 \pm 0.23$ & $0.693 \pm 0.21$ & $0.837 \pm 0.26$ & $0.67 \pm 0.2$ & $0.81 \pm 0.24$ \\
\hline Group (II) & $0.615 \pm 0.19$ & $0.532 \pm 0.15$ & $0.686 \pm 0.19$ & $0.582 \pm 0.16$ & $0.581 \pm 0.18$ & $0.521 \pm 0.14$ & $0.679 \pm 0.19$ & $0.491 \pm 0.14$ & $0.64 \pm 0.19$ & $0.53 \pm 0.16$ \\
\hline P-value & $0.4358 *$ & $0.0001 * *$ & $0.9863 *$ & $0.0003 * *$ & $0.2243 *$ & $0.0001 * *$ & $0.8097 *$ & $0.0001 * *$ & $0.5968 *$ & $0.0001 * *$ \\
\hline
\end{tabular}

M; Mean, SD; Standard Deviation, P; Probability Level

*insignificant difference

\section{MD; Mesiodistal, BL; Buccolingual}

**significant difference

TABLE (3): Depth evaluation between both groups regarding all implants and overall implants:

\begin{tabular}{|c|c|c|c|c|c|c|c|c|c|c|}
\hline & \multicolumn{10}{|c|}{ Depth $(M \pm S D)$} \\
\hline & \multicolumn{2}{|c|}{ Implant (1) } & \multicolumn{2}{|c|}{ Implant (2) } & \multicolumn{2}{|c|}{ Implant (3) } & \multicolumn{2}{|c|}{ Implant (4) } & \multicolumn{2}{|c|}{ Overall } \\
\hline & MD & BL & MD & BL & MD & BL & MD & BL & MD & BL \\
\hline Group (I) & $0.841 \pm 0.21$ & $1.01 \pm 0.31$ & $0.921 \pm 0.28$ & $0.793 \pm 0.23$ & $0.808 \pm 0.20$ & $0.843 \pm 0.26$ & $0.92 \pm 0.28$ & $0.917 \pm 0.28$ & $0.83 \pm 0.25$ & $0.87 \pm 0.26$ \\
\hline Group (II) & $0.821 \pm 0.25$ & $0.865 \pm 0.25$ & $0.808 \pm 0.20$ & $0.732 \pm 0.23$ & $0.797 \pm 0.25$ & $0.712 \pm 0.21$ & $0.836 \pm 0.21$ & $0.832 \pm 0.24$ & $0.73 \pm 0.22$ & $0.82 \pm 0.25$ \\
\hline P-value & $0.7655 *$ & $0.0811 *$ & $0.1145 *$ & $0.3630 *$ & $0.8671 *$ & $0.0610 *$ & $0.2457 *$ & $0.2647 *$ & $0.1481 *$ & $0.5005 *$ \\
\hline
\end{tabular}


TABLE (4): Angular deviation evaluation between both groups regarding all implants and overall implants:

\begin{tabular}{|c|c|c|c|c|c|c|c|c|c|c|}
\hline & \multicolumn{10}{|c|}{ Angular Deviation $(\mathrm{M} \pm \mathrm{SD})$} \\
\hline & \multicolumn{2}{|c|}{ Implant (1) } & \multicolumn{2}{|c|}{ Implant (2) } & \multicolumn{2}{|c|}{ Implant (3) } & \multicolumn{2}{|c|}{ Implant (4) } & \multicolumn{2}{|c|}{ Overall } \\
\hline & MD & BL & MD & BL & MD & BL & MD & BL & MD & BL \\
\hline Group (I) & $4.52 \pm 1.4$ & $4.86 \pm 1.46$ & $8.17 \pm 2.52$ & $7.36 \pm 2.21$ & $6.37 \pm 1.97$ & $7.98 \pm 2.4$ & $5.01 \pm 1.55$ & $4.59 \pm 1.38$ & $6.02 \pm 1.82$ & $6.20 \pm 1.87$ \\
\hline Group (II) & $2.13 \pm 0.55$ & $2.34 \pm 0.72$ & $4.78 \pm 1.24$ & $4.09 \pm 1.26$ & $3.46 \pm 0.9$ & $4.57 \pm 1.41$ & $4.21 \pm 1.09$ & $3.4 \pm 1.05$ & $3.65 \pm 1.1$ & $3.60 \pm 1.09$ \\
\hline P-value & $0.0001 * *$ & $0.0001 * *$ & $0.0001 * *$ & $0.0001 * *$ & $0.0001 * *$ & $0.0001 * *$ & $0.0443 * *$ & $0.0016 * *$ & $0.0001 * *$ & $0.0001 * *$ \\
\hline
\end{tabular}

M; Mean, SD; Standard Deviation, P; Probability Level

**significant difference

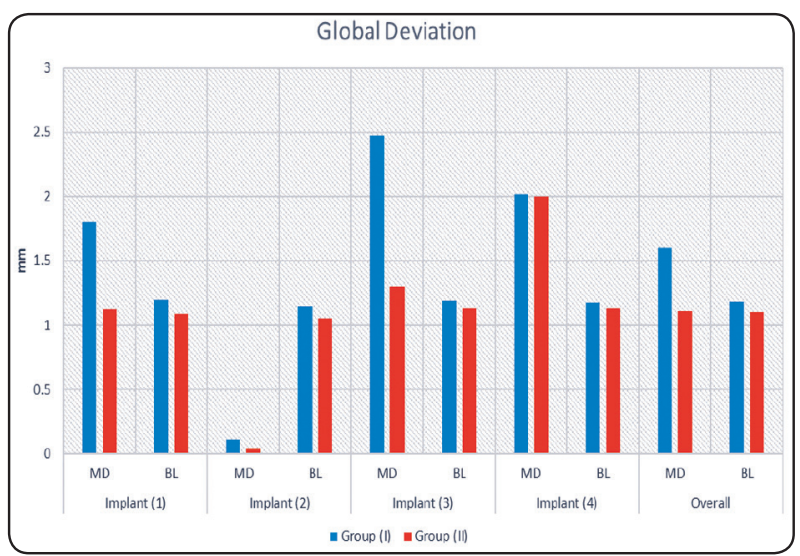

Fig. (5): Bar Chart revealing global deviation evaluation between both groups regarding all implants and overall implants

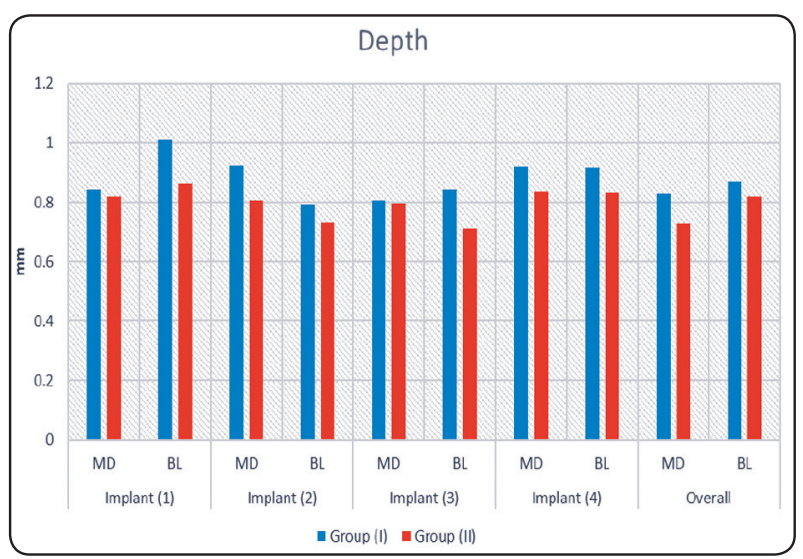

Fig. (7): Bar Chart revealing depth evaluation between both groups regarding all implants and overall implants
MD; Mesiodistal, BL; Buccolingual

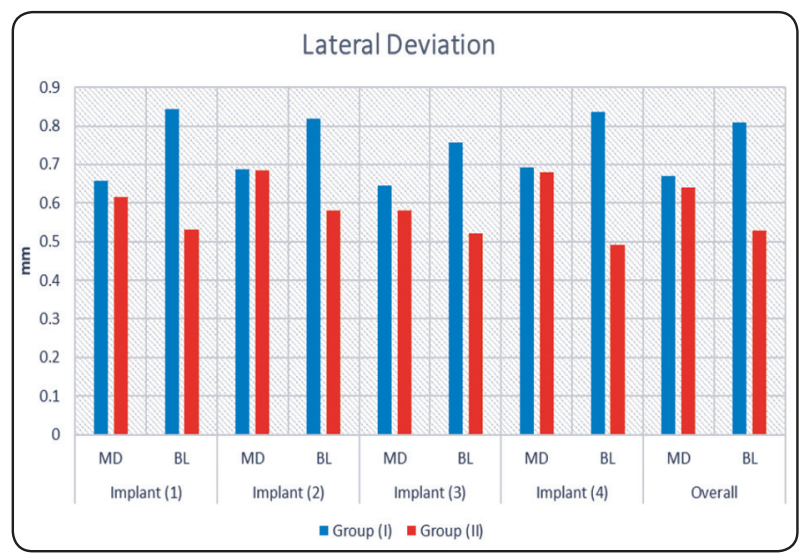

Fig. (6): Bar Chart revealing lateral deviation evaluation between both groups regarding all implants and overall implants

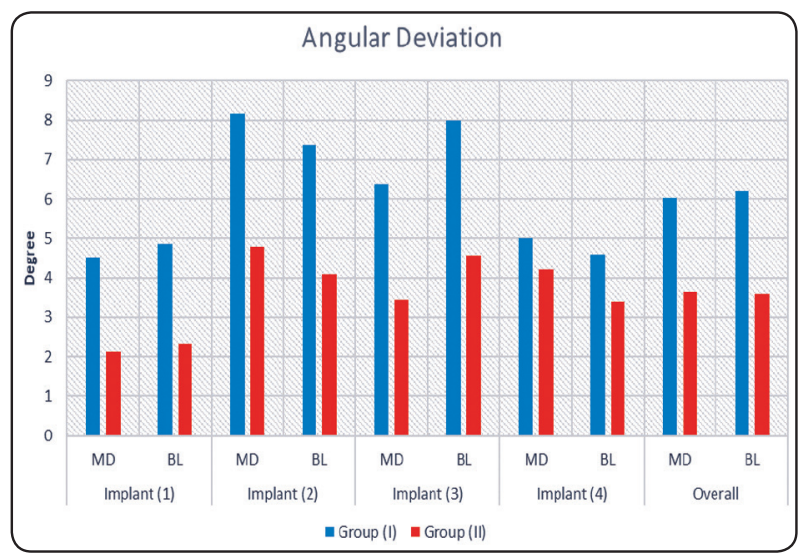

Fig. (8): Bar Chart revealing global deviation evaluation between both groups regarding all implants and overall implants 


\section{DISCUSSION}

In guided implant surgery, matching the planned site of the implant in the software with the definite site of the implant in the patient's mouth is demarcated as accuracy. It replicates the accumulation of all deviations from imaging over the transformation of data into a guide, to the improper positioning of the latter throughout the surgical procedure. There are different types of errors including error through image acquisition and data processing, error during surgical template production, error during template positioning and micromovement of the template during the drilling procedure, and mechanical error caused by tolerance of surgical instruments... ${ }^{20,35}$

All errors are cumulative, although seldom occurring. The deviation errors accumulated from every step of the procedure. As it is difficult to detect deviations that possibly occur in each step accordingly all the involved clinical factors must be considered. Issues as computer tomography (CT) scan method, type of guide, guide position, and cone beam computer tomography (CBCT) acquisition errors which includes patient movement and imaging artifacts so on were also reported in the literature..$^{20,33,35}$

A recent meta-analysis recommended leaving as a minimum safe distance; the highest value of confidence interval (CI) of the clinical studies; yet, it must be taken in consideration that some authors have obtained higher values. Consequently, regarding to global coronal deviations, it would be judicious to leave a safe distance at least $1.3 \mathrm{~mm}$ inorder to avoid any hazardous complications. Which is a very close value to the safety distance of $1.2 \mathrm{~mm}$ suggested by the European Association of Osseointegration (EAO) consensus of 2012. ${ }^{18,36}$ For global apical deviation, to leave a safety distance at least $1.7 \mathrm{~mm}$ is reasonable. Concerning angular deviation, it would be advisable to leave at least 4.7 degrees. Finally regarding implant depth, it would be prudent to leave at least $1 \mathrm{~mm}$ of margin; however the EAO consensus of 2012 recommends $0.5 \mathrm{~mm} .{ }^{37}$
In this study there was significant difference in the global deviation between group I and group II. Many authors reported that significant global deviations related to implant length can only be found in the mesio-distal direction. Furthermore others stated that global deviations can be exaggerated by the difficulty of drilling, particularly in lengthy implants, by locating long drills in restricted mouth opening ${ }^{38,39}$ This explains the deviations regarding group I (duplicated conventional complete denture incorporated with barium sulphate) in implants no. (1), (2), (3) and (4) mesiodistally and confirmed with the overall MD global deviation (1.60 \pm 0.48$)$. On the other side group II (digital tray) showed lower deviations in all implants and confirmed with the overall MD global deviations (1.11 \pm 0.34$)$.From these results the significant difference in the global deviation between groups can be attributed to the accuracy of the constructed radiographic guide and its efficiency of data acquisition in each group.

Regarding lateral deviations in our study there was significant difference between both groups regarding implant (1), (2), (3), (4) buccolingually and confirmed by overall BL lateral deviations. Upon the insertion of the distal implants through the same surgical guide, a significant greater bucco-lingual deviation at the implant platform was recognized comparable to the first distal implant. Furthermore, nearly all the bucco-lingual deviations of the second distal implant were in the direction of the lingual (left) side when operated by the right-handed surgeons, signifying a left-sided bending of the surgical guide throughout the surgical procedure. ${ }^{40}$

Many studies stated that higher lateral deviations at the apical position comparable with the coronal position, is attributable to the effect of the angular deviation. Therefore, angular deviation consequences for lateral deviation. This lateral deviation increases with the increasing distance from the guiding tube. Accordingly, the apical point of the implant is predictable to have a greater lateral de- 
viation than the coronal point. Two of the maximum decisive issues that have an important impact on the construction of planning models are the quality and further processing of imaging data, since they are the primary and fundamental steps for the following process chain. As deviations between virtual and placed implants are clearly expected even in circumstances of proper application and knowledge of state of-the-art technologies for computer-guided implant surgery, the clinician should be conscious not to overestimate advocated surgical safety by using static navigation tools. ${ }^{41}$

But the results of our study verified that digital trays provide accurate digital impression together with accurate data acquisition, precise and true $3 \mathrm{D}$ digital scan and automated registration. It is a unique tray that is especially designed for accurate digitalization for patient intraoral information.

Regarding angular deviations there was significant difference between both groups regarding implant no. (1), (2), (3), (4) and overall implants in $\mathrm{BL}$ and MD section. These results regarding group I correspond to the outcomes reported by a recent study concluding that the mean angular deviation was less than $5^{\circ}\left(4.1^{\circ}\right)$ in 236 placed implants. Which neglects the need for angled abutments in most cases when placing implants with 3D-based insertion guides. This is a great benefit for the mechanical load-bearing capacity of the implants and mostly for long lasting maintenance of the peri-implant bone ${ }^{38}$.Another review emphasized that the operators should be conscious of the angular and linear deviations up to $5^{\circ}$ and $2.3 \mathrm{~mm}$. Hence, it is significant to highlight that novice dentists should gain comprehensive training and supervision prior they place implants with $\mathrm{CAD} / \mathrm{CAM}$ surgical guides to avoid serious complications. Finally, the operators need to create a safety zone between implants and critical anatomic structures such as inferior alveolar nerve throughout selecting the accurate location as well as the proper length of the implants..$^{37,42}$ On the other hand, group II revealed an overall MD angular deviation (3.65 \pm 1.1$)$ and overall BL angular deviation $(3.60 \pm 1.09)$ which is even less than that recommended by the EAO consensus of 2012. Which stated that in case of angular deviation, it would be judicious to leave at least 4.7 degrees ${ }^{37}$

Congruence between the planned positions of the implant in the software with the actual position of the implant in the patient's mouth is an important issue. The results of this assessment indicate that group II (digital tray) proved a high degree of congruence between preoperative planning data and intraoperative results. The planned and actual values were consistent in all cases concerning implant positioning.

The main significant difference among our digital tray technique and the other clinical studies previously published is that we did not expose the patients to any additional radiation in preoperative CT or CBCT imaging for the purpose of our study. Thus, saves another scan for the patient. Only imaging of the impression in a digital tray preoperatively for accurate virtual planning for the proposed implants sites. Thus, digitalizing the impression for accurate data acquisition, 3D CT scan as well as superimposition of these 3D data for a successful prosthetically driven Implant planning and denture prosthesis.

In general, the results of our study demonstrate that it is possible to transfer a virtual implant position based on computer planning to the surgical site precisely. However, deviations were observed and require detailed analysis.

\section{CONCLUSION}

Within the limitations of this in-vitro study, the following conclusions can be listed:

1. Digital trays gave better results over conventional old denture duplication with barium sulphate incorporation. 
2. The accuracy of implant placement in both groups was within the safety margin of previous studies.

3. The total accuracy represents the sum of all cumulative errors throughout the computeraided implant placement cascade and errors can arise at different stages, the clinician should check for perfect adaptation of surgical guide before beginning of treatment, as well as during surgery.

\section{ACKNOWLEDGMENTS}

The authors have stated explicitly that there are no conflicts of interest in connection with this article.

\section{REFERENCE}

1. Widmann G, Bale RJ. Accuracy in computer-aided implant surgery--a review. Int J Oral Maxillofac Implants. 2006;21(2):305-313. http://www.ncbi.nlm.nih.gov/ pubmed/16634503

2. Adell R, Lekholm U, Rockler B, Brånemark P-I. A 15year study of osseointegrated implants in the treatment of the edentulous jaw. Int J Oral Surg. 1981;10(6):387-416. doi:10.1016/S0300-9785(81)80077-4

3. Goodacre CJ, Bernal G, Rungcharassaeng K, Kan JYK. Clinical complications with implants and implant prostheses. J Prosthet Dent. 2003;90(2):121-132. doi:10.1016/ S0022-3913(03)00212-9

4. Katsoulis J, Pazera P, Mericske-Stern R. Prosthetically Driven, Computer-Guided Implant Planning for the Edentulous Maxilla: A Model Study. Clin Implant Dent Relat Res. 2009;11(3):238-245. doi:10.1111/j.17088208.2008.00110.x

5. Kopp KC, Koslow AH, Abdo OS. Predictable implant placement with a diagnostic/surgical template and advanced radiographic imaging. J Prosthet Dent. 2003;89(6):611615. doi:10.1016/s0022-3913(03)00198-7

6. Fortin T, Champleboux G, Lormée J, Coudert JL. Precise Dental Implant Placement in Bone Using Surgical Guides in Conjunction With Medical Imaging Techniques. J Oral Implantol. 2000;26(4):300-303 . doi:10.1563/15481336(2000)026<0300:PDIPIB > 2.3.CO;2
7. Benavides E, Rios HF, Ganz SD, et al. Use of cone beam computed tomography in implant dentistry: the International Congress of Oral Implantologists consensus report. Implant Dent. 2012;21(2):78-86. doi:10.1097/ ID.0b013e31824885b5

8. Farley NE, Kennedy K, McGlumphy EA, Clelland NL. SplitMouth Comparison of the Accuracy of Computer-Generated and Conventional Surgical Guides. Int J Oral Maxillofac Implants. 2013;28(2):563-572. doi:10.11607/jomi.3025

9. Ozan O, Turkyilmaz I, Ersoy AE, McGlumphy EA, Rosenstiel SF. Clinical Accuracy of 3 Different Types of Computed Tomography-Derived Stereolithographic Surgical Guides in Implant Placement. J Oral Maxillofac Surg. 2009;67(2):394-401. doi:10.1016/j.joms.2008.09.033

10. Masri R DC. Clinical Applications of Digital Dental Technology. (Masri R, Driscoll CF, eds.). John Wiley \& Sons, Inc; 2015. doi:10.1002/9781119045564

11. Ventola CL. Medical Applications for 3D Printing: Current and Projected Uses. P T. 2014;39(10):704-711. http:// www.ncbi.nlm.nih.gov/pubmed/25336867

12. Van Noort R. The future of dental devices is digital. Dent Mater. 2012;28(1):3-12. doi:10.1016/j.dental.2011.10.014

13. Joda T, Ferrari M, Gallucci GO, Wittneben J-G, Brägger U. Digital technology in fixed implant prosthodontics. Periodontol 2000. 2017;73(1):178-192. doi:10.1111/prd.12164

14. Basten CHJ, Kois JC. The use of barium sulfate for implant templates. J Prosthet Dent. 1996;76(4):451-454. doi:10.1016/S0022-3913(96)90554-5

15. Jung RE, Schneider D, Ganeles J, et al. Computer technology applications in surgical implant dentistry: a systematic review. Int $\mathbf{J}$ Oral Maxillofac Implants. 2009;24 Suppl:92-109. http://www.ncbi.nlm.nih.gov/ pubmed/19885437

16. Spector L. Computer-Aided Dental Implant Planning. Dent Clin North Am. 2008;52(4):761-775. doi:10.1016/j. cden.2008.05.004

17. Son K, Lee W-S, Lee K-B. Effect of repeated learning for two dental CAD software programs. J Dent Rehabil Appl Sci. 2017;33(2):88-96. doi:10.14368/jdras.2017.33.2.88

18. Sicilia A, Botticelli D, Working Group 3. Computerguided implant therapy and soft- and hard-tissue aspects. The Third EAO Consensus Conference 2012. Clin Oral Implants Res. 2012;23 Suppl 6(SUPPL.6):157-161. doi:10.1111/j.1600-0501.2012.02553.x 
19. Tahmaseb A, Wu V, Wismeijer D, Coucke W, Evans C. The accuracy of static computer-aided implant surgery: A systematic review and meta-analysis. Clin Oral Implants Res. 2018;29(S16):416-435. doi:10.1111/clr.13346

20. Zhou W, Liu Z, Song L, Kuo C, Shafer DM. Clinical Factors Affecting the Accuracy of Guided Implant Surgery-A Systematic Review and Meta-analysis. J Evid Based Dent Pract. 2018;18(1):28-40. doi:10.1016/j.jebdp.2017.07.007

21. Son K, Huang M-Y, Lee K-B. A method to evaluate the accuracy of dental implant placement without postoperative radiography after computer-guided implant surgery: A dental technique. J Prosthet Dent. 2020;123(5):661-666. doi:10.1016/j.prosdent.2019.04.015

22. Martorelli M. A new approach in CT artifact removal: three cases study in maxillofacial surgery. Int $\mathrm{J}$ Interact Des Manuf. 2013;7(2):115-124. doi:10.1007/s12008-0120171-y

23. Komiyama A, Pettersson A, Hultin M, Näsström K, Klinge B. Virtually planned and template-guided implant surgery: An experimental model matching approach. Clin Oral Implants Res. 2011;22(3):308-313. doi:10.1111/j.16000501.2010.02001.x

24. Tang T, Liao L, Huang Z, Gu X, Zhang X. Accuracy of the evaluation of implant position using a completely digital registration method compared with a radiographic method. J Prosthet Dent. 2019;122(6):537-542. doi:10.1016/j. prosdent.2018.11.020

25. Schubert $\mathrm{C}$, van Langeveld MC, Donoso LA. Innovations in 3D printing: a 3D overview from optics to organs. Br J Ophthalmol. 2014;98(2):159-161. doi:10.1136/ bjophthalmol-2013-304446

26. Tahayeri A, Morgan M, Fugolin AP, et al. 3D printed versus conventionally cured provisional crown and bridge dental materials. Dent Mater. 2018;34(2):192-200. doi:10.1016/j. dental.2017.10.003

27. Whitley D, Eidson RS, Rudek I, Bencharit S. In-office fabrication of dental implant surgical guides using desktop stereolithographic printing and implant treatment planning software: A clinical report. J Prosthet Dent. 2017;118(3):256-263. doi:10.1016/j.prosdent.2016.10.017

28. Ngo TD, Kashani A, Imbalzano G, Nguyen KTQ, Hui D. Additive manufacturing ( $3 \mathrm{D}$ printing): A review of materials, methods, applications and challenges. Compos Part B Eng. 2018;143:172-196. doi:10.1016/j. compositesb.2018.02.012
29. Lin W-S, Harris BT, Pellerito J, Morton D. Fabrication of an interim complete removable dental prosthesis with an in-office digital light processing three-dimensional printer: A proof-of-concept technique. J Prosthet Dent. 2018;120(3):331-334. doi:10.1016/j.prosdent.2017.12.027

30. Sotsuka Y, Nishimoto S. Making three-dimensional mandible models using a personal three-dimensional printer. J Plast Reconstr Aesthetic Surg. 2014;67(4):576-578. doi:10.1016/j.bjps.2013.11.013

31. Deeb GR, Allen RK, Hall VP, Whitley D, Laskin DM, Bencharit S. How Accurate Are Implant Surgical Guides Produced With Desktop Stereolithographic 3-Dimentional Printers? J Oral Maxillofac Surg. 2017;75(12):2559.e12559.e8. doi:10.1016/j.joms.2017.08.001

32. Chen L, Lin W-S, Polido WD, Eckert GJ, Morton D. Accuracy, reproducibility, and dimensional stability of additively manufactured surgical templates. J Prosthet Dent. 2019;122(3):309-314. doi:10.1016/j.prosdent.2019.02.007

33. Ahmed MF, AbdelHamid AM, AlAbbasy FH. Accuracy Of Implant Placement Using Two Different Types of CAD/ CAM Surgical Guides (An Invitro Study). Alexandria Dent J. 2019;44(3):28-33. doi:10.21608/adjalexu.2019.63552

34. Lin C-C, Wu C-Z, Huang M-S, Huang C-F, Cheng H-C, Wang DP. Fully Digital Workflow for Planning Static Guided Implant Surgery: A Prospective Accuracy Study. J Clin Med. 2020;9(4):980. doi:10.3390/jcm9040980

35. Siqueira R, Chen Z, Galli M, Saleh I, Wang H, Chan H. Does a fully digital workflow improve the accuracy of computerassisted implant surgery in partially edentulous patients? A systematic review of clinical trials. Clin Implant Dent Relat Res. 2020;22(6):660-671. doi:10.1111/cid.12937

36. Hämmerle CHF, Quirynen M, Klinge B. The 3rd EAO Consensus Conference, 15-18 February 2012,Pfäffikon, Schwyz, Switzerland. Clin Oral Implants Res. 2012;23(SUPPL.6):vvi. doi:10.1111/j.1600-0501.2012.02559.x

37. Bover-Ramos F, Viña-Almunia J, Cervera-Ballester J, Peñarrocha-Diago M, García-Mira B. Accuracy of Implant Placement with Computer-Guided Surgery: A Systematic Review and Meta-Analysis Comparing Cadaver, Clinical, and In Vitro Studies. Int J Oral Maxillofac Implants. 2018;33(1):101-115. doi:10.11607/jomi.5556

38. Naziri E, Schramm A, Wilde F. Accuracy of computerassisted implant placement with insertion templates. GMS Interdiscip Plast Reconstr Surg DGPW. 2016;5:Doc15. doi:10.3205/iprs000094 
39. Sigcho López DA, García I, Da Silva Salomao G, Cruz Laganá D. Potential Deviation Factors Affecting Stereolithographic Surgical Guides: A Systematic Review. Implant Dent. 2019;28(1):68-73. doi:10.1097/ID.0000000000000853

40. Lin C-C, Ishikawa M, Maida T, et al. Stereolithographic Surgical Guide with a Combination of Tooth and Bone Support: Accuracy of Guided Implant Surgery in Distal Extension Situation. J Clin Med. 2020;9(3):709. doi:10.3390/jcm9030709
41. Stübinger S, Buitrago-Tellez C, Cantelmi G. Deviations between Placed and Planned Implant Positions: An Accuracy Pilot Study of Skeletally Supported Stereolithographic Surgical Templates. Clin Implant Dent Relat Res. 2014;16(4):540-551. doi:10.1111/cid.12019

42. Unsal G, Turkyilmaz I, Lakhia S. Advantages and limitations of implant surgery with CAD/CAM surgical guides: A literature review. J Clin Exp Dent. 2020;12(4):e409e417. doi:10.4317/jced.55871 